\title{
Categorization of Respiratory Signal using ANN and SVM Based on Feature Extraction Algorithm
}

\author{
T. Jayasri ${ }^{1 *}$ and M. Hemalatha ${ }^{2}$ \\ 'Embedded Systems, School of Computing, SASTRA University, Thanjavur, TamilNadu, India; \\ jayasri5591@gmail.com \\ ${ }^{2}$ Senior Assistant Professor, School of Computing, SASTRA University, Thanjavur, TamilNadu, India; \\ hemalatha@it.sastra.edu
}

\begin{abstract}
Sleep apnea is a dishevelment that causes interruption in breath or shoal of the respiration. The respiratory signal is classified into three states such as normal respiration, motion artifacts, and sleep apnea and it is obtained from a physionet. Firstly, using a second order auto regressive modeling, an algorithm is developed to attain the energy and frequency parameters of the signal and then the signal is classified with threshold based manual classification into any of the above taxonomy. In addition to this dataset, MLP is trained with a back propagation learning algorithm that results in reduced time, iterations and errors. Consequently, the training of SVM, a binary classifier used to solve multiple class problems is done with the same data set and classification is made to reduce overall errors. The overall efficiency of the above techniques is compared.
\end{abstract}

Keywords: Feature Extraction, Autoregressive Model, Burgs Method, Multilayer Perceptron, Back Propagation Learning Algorithm, Support Vector Machine.

\section{Introduction and Literature Survey}

Sleep apnea diagnosis is essential because as associated with health hazards such as hypertension, arrhythmia, heart failure, stroke, diabetes, depression and reduced working efficiency. It leads to examples of leap of faith and results in a distorted view of any situation. The primary aim is to develop an efficient algorithm for classification of respiratory signals and thereby detect sleep apnea. For instance this can be obtained by a simple method for respiratory signal classification using a MATLAB coding and by using neural networks and SVM. Most importantly, one of the main challenges in this work is to render concepts and technology from the fields of bio signals and human physiology into a single classifying algorithm. Moreover, analysis of the signal, research and development of efficient algorithm are the major contribution to this work. On the other hand respiration is measured for medical examinations like sleep analysis, pulmonary functions, pulmonary screening, stress test, pediatric screening, and pediatric monitoring-Sudden Infant Death Syndrome (SIDS). In general, the respiratory signal may be categorized into normal, sleep apnea and motion artifacts and one cannot deny that more importance is given for detecting sleep apnea because it leads to an irregular heartbeat, high blood pressure, heart attack, etc... Specifically, respiration is characterized by the presence of a certain rhythm and the presence of some energy level in the signal. As a result, apnea is characterized by the absence of energy as well as a lack of rhythm. Motion Artifact is typically characterized by a sudden increase in the amplitude of the signal. The Greek originated word apnea meaning "devoid of wind" usually occur in sleep. If regular breathing reduced to $25 \%$

* Corresponding author:

T. Jayasri (jayasri5591@gmail.com) 
or stopped absolutely over nearly 10 seconds then it is apnea. Another elucidation of apnea forms a $4 \%$ descent in blood oxygen level [1]. Apnea index calculated as the ratio of number of apnea to the number of hours slept. The risk of apnea increases with increase in apnea index. Figure 1 here shows a model of respiratory signal consisting of apnea, normal, and artifacts. Sleep apnea being probably a hazardous situation appears in all generation. The aspiration of this work is to offer a possibility of steadily monitoring respiration using non-invasive biomedical signals. The signal of primary interest is Respiratory signal. Respiratory signals are convenient to measure by wearing sensors without the requirement to place electrodes. The FPGA can also be employed for implementing respiratory signal classification [2]. In this work autoregressive modeling (AR) and modified zero-crossing algorithm used for classification and provides good results for normal respiration and apnea but disagreements encountered in motion artifacts detection. Thus an algorithm is used for automatic classification of respiratory signal which uses the features obtained from autoregressive modeling [3] and classify them based on threshold value into normal, apnea and motion artifacts. Admittedly, this algorithm utilizes autoregressive modeling and zero-crossing [4] for classification of respiratory signal into normal respiration, apnea and respiration with artifacts. Braunwald.J et al., a have detailed the effects of respiratory system disorder in sleep apnea[5].

\section{Feature Extraction}

The Massachusetts Institute of Technology-Beth Israel Hospital (MIT-BIH) Polysomnography database is a portion of the Physionet archive about biomedical signal databases. The MIT-BIH Polysomnography Database comprises 16 continuous Polysomnography recordings from male subjects all of whom suffer from sleep apnea. In this work respiratory signal's features were extracted by using classification algorithm and are compared against threshold

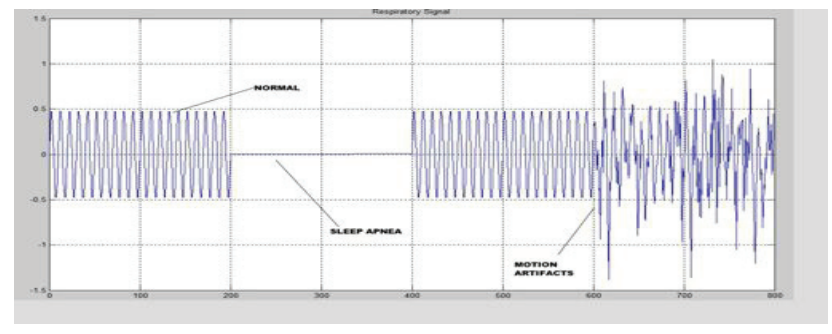

Figure 1. Respiratory signal. values for apnea detection. The fundamental extracted features [3] of respiratory signals are given below.

\subsection{Energy Index (EI)}

The maximum amount of energy resides in the signal is referred as EI. The Energy index is computed using the following equation:

$$
\frac{1}{\mathrm{~N}} \sum_{\mathrm{n}=0}^{\mathrm{N}-1}\left|\mathrm{x}_{\mathrm{n}}\right|_{2}
$$

The total energy is given by

$$
\varepsilon_{x} \triangleq \sum_{\mathrm{n}=0}^{\mathrm{N}-1}\left|\mathrm{x}_{\mathrm{n}}\right|_{2}
$$

$\varepsilon_{x}$ is defined without considering constant scale factor such as sampling interval or wave impedance. The average power is define as the energy present per sample,

$$
\mathrm{Px} \triangleq \frac{\varepsilon_{\mathrm{x}}}{\mathrm{N}}=\frac{1}{\mathrm{~N}} \sum_{\mathrm{n}=0}^{\mathrm{N}-1}\left|\mathrm{x}_{\mathrm{n}}\right|^{2}
$$

\subsection{Respiration Rate (FZX)}

Respiratory rate is defined as the number of breaths per minute. At rest for every minute $12-18$ breath is encountered as respiratory rate for normal adult [6]. The number of times a signal crisscross over reference line is denoted as respiratory rate.

$$
\mathrm{FZX}=\sqrt{E I}
$$

\subsection{Dominant Frequency (FAR)}

The Dominant Frequency is the frequency which is occurring most often.

Dominant frequency (Freq AR) $=$ Freq AR $=\frac{F_{s}}{2 \pi} \arctan \frac{a_{1}}{a_{2}}$

The sampling frequency (Fs) of $250 \mathrm{~Hz}$ was used for analysis. In addition the average energy of signal is also calculated.

\subsection{Strength of Dominant Frequency (STR)}

The second auxiliary polynomial built from AR coefficient and position of set of complex conjugate roots used to estimate FAR and STR. Where FAR=sampling frequency*angle/360 and STR=extension from the base. FAR and STR essentially indicates dominant frequency and its power level, as power spectrum does. The classification of the signal is based on derived parameters above. 
Signal strength $(\mathrm{MagAR})=\mathrm{MagAR}=\sqrt{a_{1}^{2}+a_{2}^{2}}$

\subsection{Thresholds $[3,2]$}

The computed features are compared with threshold values for classification. The thresholds required are determined automatically by the following procedure.

For real time respiratory signal, the threshold values obtained are

\section{Computation for AR Coefficients}

The autoregressive model (AR) is linear prediction formula which predicts an output based on the previous outputs and inputs. The definition used is,

$$
y_{m}=\sum_{i=0}^{m} a_{i} x_{m-i}+\epsilon_{m}
$$

where $a_{i}$ is the autoregression coefficients, $x_{m}$ is the series under inquiry and $\mathrm{n}$ is the order of the filter which is less than the series length. $\in$ is taken as white Gaussian noise. The current term in the linear sequence estimated using weighted sum of preceding term and the weights represents the coefficient of AR model. AR modeling is used because each type of process (MA, AR and ARMA) converted to other types. By solving linear set of equations AR model can be found. It has much better frequency resolution. An

Table 1. Threshold values used for classification of respiratory signal.

\begin{tabular}{ll}
\hline \multicolumn{1}{c}{ FEATURES } & \multicolumn{1}{c}{ RANGE } \\
\hline EI_MIN \& EI_MAX & $33 \%$ \& 150\% of average energy \\
FZX_MIN \& FZX_MAX & $0.2 \mathrm{~Hz} \&$ 0.7 Hz of average rate \\
FAR_MIN \& FAR_MAX & $\begin{array}{l}50 \% \text { \& 150\% of dominant } \\
\text { frequency }\end{array}$ \\
STR_MIN \& STR_MAX & $\begin{array}{l}\text { 75\% \& 95\% of average } \\
\text { strength }\end{array}$ \\
\hline
\end{tabular}

Table 2. Threshold values for a real time respiratory signal.

\begin{tabular}{ll}
\hline \multicolumn{1}{c}{ FEATURES } & \multicolumn{1}{c}{ RANGE } \\
\hline EI_MIN \& EI_MAX & $33 \%$ \& $150 \%$ of 0.0379 \\
FZX_MIN \& FZX_MAX & $0.2 \mathrm{~Hz} \& 0.7 \mathrm{~Hz}$ of 0.1948 \\
FAR_MIN \& FAR_MAX & $50 \%$ \& $150 \%$ of -41.4606 \\
STR_MIN \& STR_MAX & $75 \%$ \& $95 \%$ of 1.9822 \\
\hline
\end{tabular}

AR model possesses fewer coefficients than MA model. Techniques like Least squares method and Burg method are available for computing AR Coefficients. Least square method which is based on Yule-Walker equation give rise to poor estimation of parameter even for data samples of reduced size and unstable model. Therefore, Burgs method is used.

\subsection{Burgs Method}

A collection of all-pole prototype parameters which reduce the total prediction errors are found using Burg algorithm [7]. Degradation is performed in terms of reflection coefficient for assuring a stable model. Autoregressive parameters calculated using Burg method are perfect than autocorrelation because the data is not subjected to any window [8]. The advantage of Burg's technique is Stable AR model, computationally it is efficient method and having high frequency resolution.

The AR coefficients using Burgs algorithm can be calculated as,

- Select the requested number of coefficients, c.

- Set $\mathrm{A} 0=[1]$.

- Using $\mathrm{f}_{\mathrm{p}}(\mathrm{m})=\sum_{i=0}^{p} a_{i} x_{m-i}$ and $\mathrm{b}_{\mathrm{p}}(\mathrm{m})=\sum_{i=0}^{p} a_{i} x_{m+i}$,

- Initialize all $\mathrm{f}_{0}(\mathrm{~m})=\mathrm{b}_{0}(\mathrm{~m})=\mathrm{x}_{\mathrm{m}}$.

- Using

$$
\begin{gathered}
\mathrm{F}_{\mathrm{p}}=\sum_{(m=p)}^{M}\left(a_{(0)} x_{m}+\sum_{(i=1)}^{p} a_{i} x_{(m-i)}\right)^{2}=\sum_{m=p}^{M}\left(\sum_{i=0}^{p} a_{i} x_{m-i}\right)_{2} \\
=\sum_{m=p}^{M}\left(f_{p}(m)\right)_{2}
\end{gathered}
$$

and

- $\mathrm{B}_{\mathrm{p}}=\sum_{(m=p)}^{(M-p)}\left(a_{(0)} x_{(m)}+\sum_{(i=1)}^{p} a_{i} x_{(m+i)}\right)^{2}=\sum_{m=0}^{M-p}\left(\sum_{i=0}^{p} a_{i} x_{m-i}\right)_{2}$

$$
=\sum_{m=0}^{M-p}\left(b_{p}(m)\right)_{2}
$$

- Compute $\mathrm{F}_{0}$ and $\mathrm{B}_{0}$

- Using $\mathrm{D}_{\mathrm{p}}=\mathrm{F}_{\mathrm{p}}-\mathrm{f}_{\mathrm{p}}(\mathrm{p})^{2}+\mathrm{B}_{\mathrm{p}}-\mathrm{b}_{\mathrm{p}}(\mathrm{M}-\mathrm{p})^{2}$, Compute $\mathrm{D}_{0}$.

- P starting from 0 to $\mathrm{c}-1$, compute the following:

- Compute $\mu=\frac{-2 \sum_{m=0}^{M-p-1} f_{p}(m+p+1) b_{p}(m)}{D_{p}}$

- Where $\mu$ is the reflection coefficient

- Update $\mathrm{A}_{\mathrm{p}+1}$ using $\mathrm{a}_{\mathrm{m}}{ }^{\prime}=\mathrm{a}_{\mathrm{m}}+\mu \mathrm{a}_{\mathrm{p}+1-\mathrm{m}}$ 
- Update $\left(\mathrm{f}_{\mathrm{p}+1}(\mathrm{~m})\right)_{\mathrm{m}}$, where $\mathrm{m}^{\in}[\mathrm{p}+1, \mathrm{M}]$

Using $\mathrm{f}_{\mathrm{p}+1}(\mathrm{~m})=\mathrm{f}_{\mathrm{p}}(\mathrm{m})+\mu \mathrm{b}_{\mathrm{p}}(\mathrm{x})$, where $\mathrm{x}=(\mathrm{m}-\mathrm{p}-1)$

- Update $\left(\mathrm{b}_{\mathrm{p}+1}(\mathrm{~m})\right)_{\mathrm{m}}$, where $\mathrm{m}^{\in}[0, \mathrm{M}-\mathrm{p}-1]$

Using $b_{p+1}(m)=b_{p}(m)+\mu f_{p}(y)$, where $y=(m+p+1)$

- Update $\mathrm{D}_{\mathrm{p}}$ using,

$$
D_{p}=\left(1-\mu^{2}\right) D_{p}-f_{p+1}(p+1)^{2}-b_{p+1}(M-p-1)^{2}
$$

\section{Automatic Classification Algorithm}

The respiratory signal is analyzed and classified using the following procedure,

a. Human respiratory signal is taken as samples

b. The respiratory signal is classified

c. The samples of respiratory signals is given as input to the classifying algorithm

d. Mathematical model is formed for the input respiratory signal using second order auto regressive modeling

e. Four features are extracted from the signal for determining the threshold values

f. In these four features, energy index and respiratory rate are calculated using (a) and (d)

g. The next two features, dominant frequency and strength of dominant frequency are calculated using Burgs algorithm as mentioned in (e) and (f)

h. Threshold values are calculated using the above obtained features in reference with threshold table (i)

i. For each cycle of the input respiratory signal, again the four features are extracted and compared with the threshold values

j. Based on this comparison the respiratory signal is classified.

\section{Feed Forward Neural Networks}

Multi Layer Perceptron (MLP) $[9,10]$ being feed forward, fully connected neural network has input, output and one or hidden layer with nodes acting as non-linear activation function except the input nodes to solve non-linear separable problems and maps a collection of input to a collection of applicable output. Figure 2 shows the confusion matrix obtained when the dataset is given to the coding. The overall efficiency obtained using Feed forward neural network was $96 \%$.

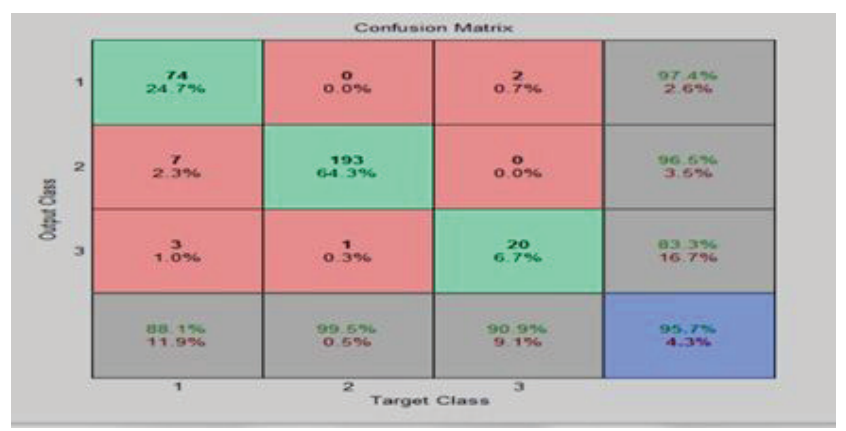

Figure 2. Confusion matrix for MLP classification.

\section{Support Vector Machine (SVM)}

A Support Vector Machine [12] is a supervised machine learning method appropriate for categorization and retrogression. It is a non-probabilistic binary linear classifier, developed from Statistical Learning Theory. It has high accuracy rate and ability to deal with high dimensional data. The support vector machine performs pattern recognition between two point classes by Support Vectors (SV). The methods are organized into two approaches, in which one tries to built SVM with multiclass called single machine approach and other breaks down the multiclass into various binary subsidiary problems to form a normal SVM for every single binary subsidiary problems called divide and conquer approach. SVM uses kernel function to estimate dot-product in high dimensional feature region and also employ a classifier which has no representation for apparent stable dimensional vector region. Linear classifier methods used to produce non-linear decision boundaries lays a major advantage.

\subsection{Program Routine}

SVM is basically a binary classifier. Since the problem taken is a multiclass problem, initially the problem is converted into a binary classification as shown in Figure 4.

- Load the dataset and classes file.

- Check the number of classes, if it is greater than 2, display it as an multiclass problem.

- Initialize classes $=0$ and no. of iteration $=1$.

- Check the following condition:

- Classes $\sim=1$ and itr $<=$ length (u)

- If the above condition is satisfied, assign a new class which contains only one class $(1,0)$. 
- The training and testing samples are randomly selected.

- Then the SVM is trained using svmtrain command and then the classification is made by using svmclassify command.

\subsection{Flowchart}

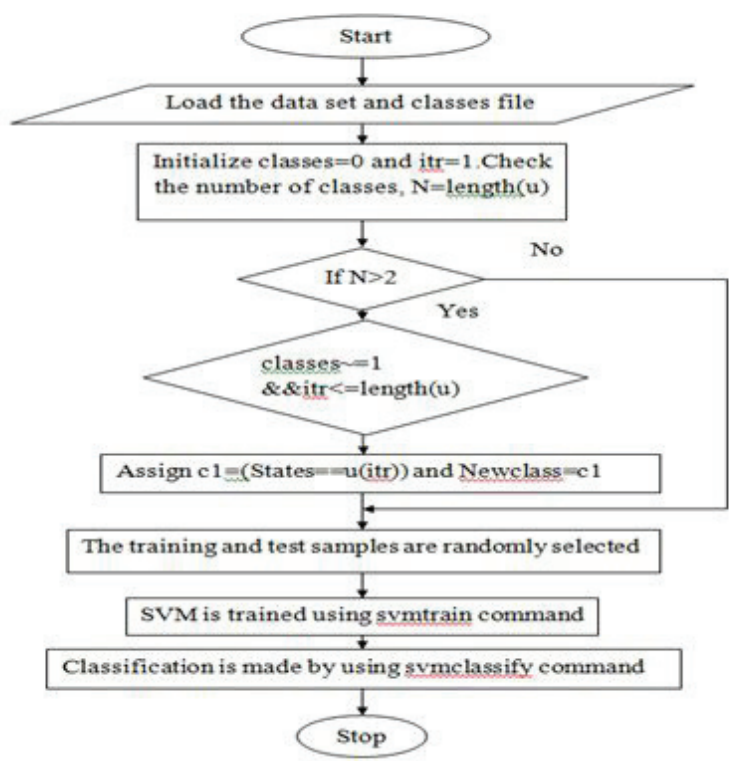

Figure 4. Flowchart for classification using SVM.

\subsection{Results}

When the dataset is given to the coding, Figure 5 here shows the hyperplane separating the classes.

The overall efficiency obtained using SVM was $97 \%$. Figure 6 here shows the confusion matrix and Figure 7 here shows the ROC plots of SVM.

\section{Discussion and Conclusion}

In conclusion, the taxonomy starts from extracting the features such as energy index, respiration rate, dominant frequency and its strength of the signal through auto regressive modeling and other techniques. Ultimately, the parameters of AR are calculated by using burgs algorithm. Therefore, these features are then compared to the predetermined threshold values to classify the respiratory signal. It considerably strengthens due to the all the mentioned relevant facts and hence the signal is classified successfully with the developed algorithm. But, the bio-signals are nonlinear signals; hence another coding is developed for signal classification in neural networks and SVM. The data set is

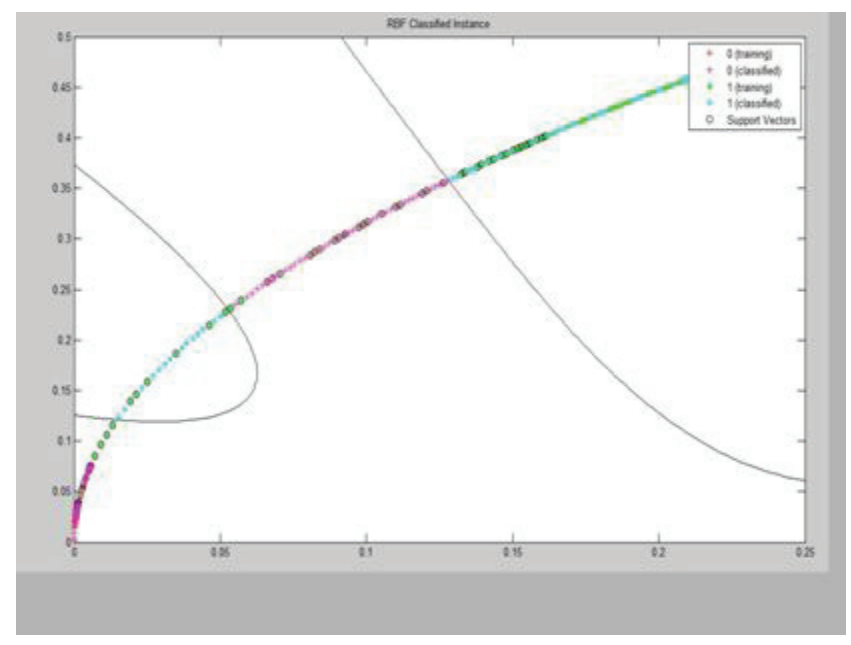

Figure 5. Classification of classes by hyperplane in SVM.

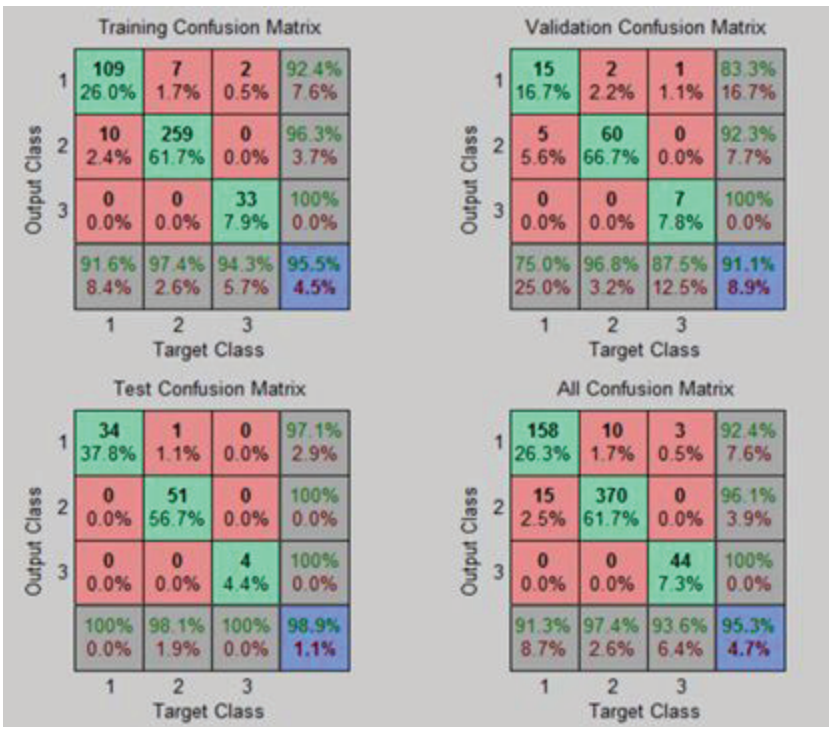

Figure 6. Confusion Matrix for SVM classification.

created with four extracted features determined in threshold based manual classification and is given as abstract to the neural networks and SVM. In closing, it is trained with it and signal classification is successfully made. The efficiency of classification is determined for both techniques. Because of the increasing awareness of the dangers caused by sleep apnea it is always profitable to consider the above reasoning for better healthcare.

\section{References}

1. Guyton C, and John E H (1998). Text book of medical Physiology, $9^{\text {th }}$ Edn., Saunders, Company publications. 


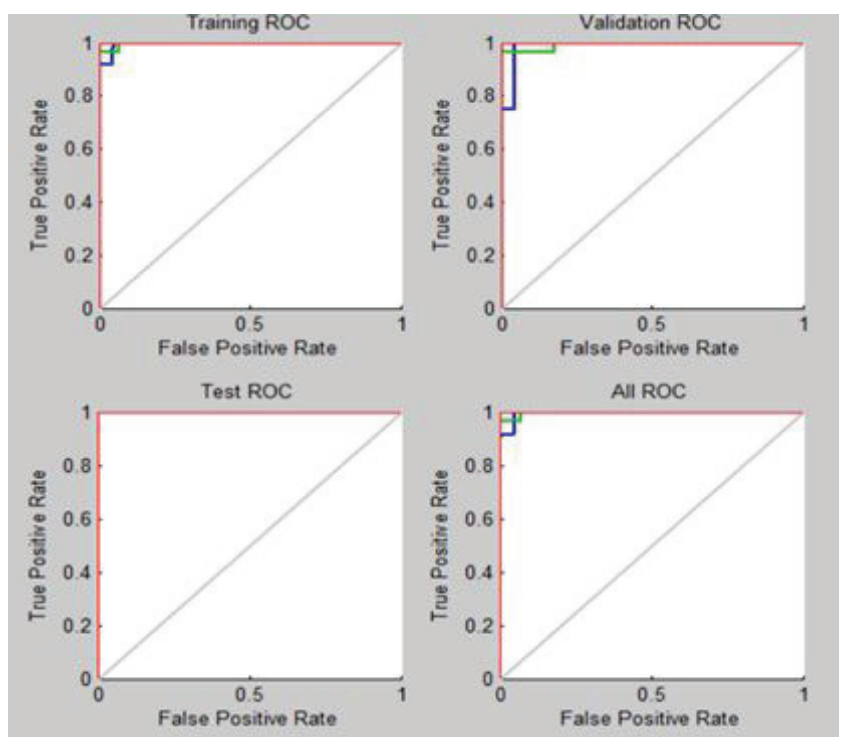

Figure 7. ROC plot for SVM classification.

2. Marinkovic B, Gillete $M$ et al. (2005). FPGA implementation of respiration signal classification using a soft-core processor.

3. Bronzino J D, and Ning T (1989). Automatic classification of respiratory signals, Conference of the IEEE Engineering in Proceedings of the Annual International, vol 2, 669-670.
4. Nepal K, Trinity Coll et al. (2002). Apnea detection and respiration rate estimation through parametric modeling, Proceedings of the IEEE 28th Annual Northeast Bioengineering Conference, 277-278.

5. Braunwald J, Fauci A et al. (2008). Harrisons Principles of Internal Medicine, $17^{\text {th }}$ Edn., Disease of respiratory system, Tata McGraw Hill Publications, 2186-2188.

6. Tortora G J, and Anagnostakos N P (1990). Principles of Anatomy and Physiology, $6^{\text {th }}$ Edn., The respiratory system, New York: Harper-Collins, 874-920.

7. Claerbout J (1997). Burg Spectral Estimation, Available from: http://sepwww.stanford.edu/sep/prof/fgdp/c7/paper_html/ node3.html

8. Hayes M H (2002). Statistical Digital Signal Processing and Modelling, Signal modelling, Wileys and Sons, 188-198.

9. Cybenko C (1989). Approximations by superpositions of a sigmoidal Function, Mathematics of Control, Signals and Systems, vol 5(4), 455.

10. A brief history of Neural Networks, Available from: www. dtreg.com

11. Brijeshverma (1997). Fast Training of Multilayer Perceptron, IEEE Transactions on Neural Networks, vol 8(6), 1314-1320.

12. Gunn S R (1998). Support vector machine for classification and regression, Technical Report, University of Southampton. 